\title{
Validation of Version 3.0 of the Breast Cancer Genetics Referral Screening Tool (B-RST ${ }^{\mathrm{TM}}$ )
}

\author{
Cecelia Bellcross, PhD, MS ${ }^{1}$, April Hermstad, $\mathrm{MPH}^{2}$, Christine Tallo, $\mathrm{MMSc}^{3}$ and \\ Christine Stanislaw, $\mathrm{MS}^{3}$
}

\begin{abstract}
Purpose: Despite increased awareness of hereditary breast and ovarian cancer among clinicians and the public, many $B R C A 1 / 2$ mutation carriers remain unaware of their risk status. The Breast Cancer Genetics Referral Screening Tool (B-RST ${ }^{\mathrm{m} *}$ ) was created and validated to easily identify individuals at increased risk for hereditary breast and ovarian cancer for referral to cancer genetics services. The purpose of this study was to revise B-RST ${ }^{\text {Tx }}$ to maximize sensitivity against $B R C A 1 / 2$ mutation status.
\end{abstract}

Methods: We analyzed pedigrees of 277 individuals who had undergone BRCA1/2 testing to determine modifications to the B$\mathrm{RST}^{\mathrm{Tx}} 2.0$ algorithm that would maximize sensitivity for mutations, while maintaining simplicity. We used McNemar's chi-square test to compare validation measures between the revised version (3.0) and the 2.0 version.

Results: Algorithmic changes made to B-RST 2.0 increased the sensitivity against $B R C A 1 / 2$ mutation analysis from 71.1 to $94.0 \%$ $(P<0.0001)$. While specificity decreased, all screen-positive individuals were appropriate for cancer genetics referral, the primary purpose of the tool.

Conclusion: Despite calls for $B R C A 1 / 2$ population screening, there remains a critical need to identify those most at risk who should receive cancer genetics services. B-RST ${ }^{\mathrm{Tm}}$ version 3.0 demonstrates high sensitivity for $B R C A 1 / 2$ mutations, yet remains a simple and quick screening tool for at-risk individuals.

Genetics in Medicine (2019) 21:181-184; https://doi.org/10.1038/ s41436-018-0020-x

Key Words: BRCA1/2; Screening tools; Genetic counseling; Hereditary breast; Ovarian cancer

\section{INTRODUCTION}

Mutations in the BRCA1 and BRCA2 genes (BRCA1/2) remain the best described and most common cause of highly penetrant hereditary breast and ovarian cancer (HBOC). The general population frequency of $B R C A 1 / 2$ mutations is approximately $1 / 200-1 / 400$, and up to $1 / 40$ among individuals of Ashkenazi Jewish descent. ${ }^{1,2}$ Due to numerous publications and celebrity media attention, awareness of HBOC among the healthcare community and public has increased over the past decade. However, the lack of identification of mutation carriers remains a concern, particularly as enhanced screening and preventative surgery have solidly demonstrated reductions in morbidity and mortality. ${ }^{3-5}$ It has been estimated that only $6 \%$ of $B R C A$ mutation carriers in the general population have been identified, ${ }^{6}$ and a recent study found that fewer than onein-five at-risk breast or ovarian cancer patients have undergone genetic testing. ${ }^{7}$

In 2005, the United States Preventive Services Task Force (USPSTF) recommended that women with family histories suggestive of a BRCA1/2 mutation be referred for genetic counseling. ${ }^{8}$ They noted the need to develop screening tools to assist clinicians in identifying women who would benefit from genetic counseling for HBOC. In their 2014 update, the USPSTF recommended that providers use one of several validated screening tools to identify woman at risk for BRCA1/2 mutations for referral to genetic counseling and, if appropriate, testing. ${ }^{9}$ The Referral Screening Tool-a precursor to the $\mathrm{B}-\mathrm{RST}^{\mathrm{m}}$ - was one of five validated tools suggested for this purpose.

The paper-based Referral Screening Tool was originally developed and validated in $2007 .{ }^{10} \mathrm{~A}$ revised web-based version-the Breast Cancer Genetics Referral Screening Tool $\left(B-R^{2} T^{m}\right)$ - was launched in 2010 and mentioned in the USPSTF recommendation. ${ }^{9,11}$ Both versions were validated against analysis of four-generation cancer pedigrees using BRCAPRO, BOADICEA, Myriad II, and the Family History Assessment Tool, with a $\geq 10 \% B R C A 1 / 2$ mutation probability or a Family History Assessment Tool score of 10 as the true measure of "high" risk. ${ }^{10,11}$ The second version (B-RST ${ }^{\text {mi }} 2.0$ ) demonstrated an overall sensitivity and specificity of 89.4 and $91.5 \%$ respectively, with a sensitivity of $100 \%$ compared with the most accurate models, BRCAPRO and BOADICEA ${ }^{11}$. ${\mathrm{B}-R S T^{\mathrm{m} t}}$ is not designed to provide a probability for a

\footnotetext{
${ }^{1}$ Department of Human Genetics, Emory University School of Medicine, Atlanta, GA, USA; ${ }^{2}$ Rollins School of Public Health, Emory University, Atlanta, GA, USA; ${ }^{3}$ Department of Human Genetics, Emory Healthcare, Winship Cancer Institute, Emory University School of Medicine, Atlanta, GA, USA. Correspondence: Cecelia Bellcross (cecelia.a. bellcross@emory.edu)
} 
$B R C A 1 / 2$ mutation, but rather categorizes the individual into one of three groups indicating the likelihood of HBOC, as well as the level of risk for breast and ovarian cancer based on family history. Screen "positive" indicates an increased risk for HBOC of 5-10\% or greater. Screen "negative-moderate risk" indicates a low risk for HBOC, but some increased risk for breast cancer, while screen "negative-average risk" indicates HBOC is very unlikely and the individual is at average risk for breast and ovarian cancer. Individuals who screen positive on

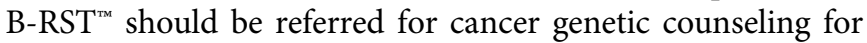
more in-depth risk assessment and discussion of genetic testing options.

The criteria for consideration of genetic counseling and testing for $B R C A 1 / 2$ have become less stringent over the past few years, with a $\geq 10 \%$ mutation probability no longer considered the minimum threshold. ${ }^{12,13}$ This is also reflected in the changes that have occurred in the National Comprehensive Cancer Network (NCCN) Guidelines ${ }^{\oplus}{ }^{14,15}$ In addition, reduction in the cost of genetic testing, and identification of BRCA mutations in lower-risk cohorts, has led some to advocate population-based testing. ${ }^{16}$ However, there remain significant logistical concerns with this approach, including lack of infrastructure to provide appropriate pre- and posttest genetic counseling, uncertainty regarding the penetrance of mutations identified in this way, and the large number of variants of uncertain significance expected. ${ }^{17}$ Until such issues are resolved, there remains a need to identify those most likely to benefit from genetic counseling and testing for HBOC, especially given the limited availability of these resources and healthcare dollars.

Multiple models exist to predict the likelihood of developing breast cancer and/or hereditary risk. ${ }^{18}$ The NCCN Guidelines ${ }^{\circledR}$, revised annually, provide criteria for both consideration of genetic counseling and testing. ${ }^{15}$ However, these options do not allow for either simple self-identification or quick screening by clinicians. Screening tools thus continue to play an important role in maximizing population-based identification of individuals who would benefit from cancer genetics services.

To address the shift toward less stringent referral criteria, we evaluated the accuracy of B-RST ${ }^{\mathrm{rm}} 2.0$ against families tested for $B R C A 1 / 2$ mutations, rather than a $\geq 10 \%$ mutation probability. Based on these data, we adapted the B-RST ${ }^{\mathrm{mm}}$ algorithm (3.0) to maximize sensitivity, while maintaining simplicity and reasonable specificity.

\section{MATERIALS AND METHODS}

We identified patients seen for genetic counseling for HBOC through Emory Healthcare (2011-2013) using the following International Classification of Diseases, Ninth Revision codes: V16.3, Family history of breast cancer; V16.41, Family history of ovarian cancer; V10.3, Personal history of malignant neoplasm of breast; V10.43, History of ovarian malignancy; 183.0, Malignant neoplasm, ovary; and 174.9, Malignant neoplasm, breast not otherwise specified. Patients seen from 2008-2013 at the Winship Cancer Institute for family history of breast cancer were identified via a clinic database. Through retrospective chart review, we obtained full three-generation cancer pedigrees and genetic test results. Only patients who had undergone BRCA1/2 testing were included in the analysis. Patients seen for known mutation follow-up or a variant of uncertain significance were excluded. For patients seen due to a previously identified familial mutation, the pedigree was evaluated based on a first-degree relative of an individual who had tested positive. This study was approved by the Emory University Institutional Review Board.

We analyzed each pedigree using B-RST ${ }^{\mathrm{Tm}} 2.0$ to determine the screening results (that is, positive, negative-moderate risk, or negative-average risk). Pedigrees with a $B R C A 1 / 2$ mutation who screened negative on B-RST ${ }^{\mathrm{m}} 2.0$ were evaluated to determine the specific family history parameters not included in B-RST ${ }^{\mathrm{m} m}$ 2.0. We explored which specific combinations of additional or revised family history features maximized sensitivity, but retained the simplicity of the tool. Features that did not significantly change the sensitivity were not added to the final algorithm. BRCA mutation-negative pedigrees were also analyzed to determine whether they were screen positive or negative on various iterations of the revised algorithms. BRCAPRO probabilities of pedigrees who were mutation negative but screen positive on $\mathrm{B}^{-\mathrm{RST}^{\mathrm{mm}}} 2.0$ and 3.0 were calculated. ${ }^{19}$

We evaluated the sensitivity, specificity, and positive and negative predictive values (PPV and NPV) of B-RST ${ }^{\mathrm{Tm}} 2.0$ and the final B-RST ${ }^{\mathrm{m}} \quad 3.0$ algorithm. We used a two-sided McNemar's chi-square test for correlated proportions to compare sensitivity and specificity between B-RST ${ }^{\mathrm{TM}} 2.0$ and 3.0. We chose not to report the overall accuracy using area under the curve, as this measure assumes equal importance of sensitivity and specificity and our goal was to maximize the former. We used $t$-tests to compare BRCAPRO probabilities. A $P$ value of less than 0.05 was considered significant.

\section{RESULTS}

Charts were reviewed for 277 patients, of which $83(30 \%)$ were positive for a $B R C A 1 / 2$ mutation in themselves or a firstdegree relative. Table 1 illustrates the $B-R_{S T}{ }^{\mathrm{mm}} 2.0$ vs. 3.0 screening results. We found a shift of negative-moderate risk to screen positive, with significantly more patients screening positive using B-RST ${ }^{\mathrm{TM}} 3.0(P<0.0001)$. There was

Table 1 Cohort screening results of B-RSTTM 2.0 vs. 3.0

\begin{tabular}{|c|c|c|c|}
\hline $\begin{array}{l}\text { B-RST }{ }^{\mathrm{TM}} \text { result } \\
(n=277)\end{array}$ & $\begin{array}{l}\text { B-RST'M } \\
2.0, \\
n(\%)\end{array}$ & $\begin{array}{l}\text { B-RST'M } \\
3.0 \\
n(\%)\end{array}$ & $P$ value \\
\hline Screen positive & $150(54.2)$ & $215(77.6)$ & $<0.0001$ \\
\hline $\begin{array}{l}\text { Screen negative (moderate } \\
\text { risk) }\end{array}$ & $111(40.1)$ & $51(18.4)$ & $<0.0001$ \\
\hline Screen negative (average risk) & $16(5.8)$ & $11(4.0)$ & 0.06 \\
\hline
\end{tabular}


Table 2 Validation measures of B-RSTTM 2.0 vs. 3.0

Validation measure $n=277$ Mut $+=83(30.0 \%)$

B-RST'M 2.0

$(95 \% \mathrm{Cl})$

\begin{tabular}{l}
\hline Sensitivity \\
B-RSTTM +/Mut \\
Specificity \\
B-RSTTM_/Mut \\
PPV
\end{tabular}

NPV

$59 / 83=71.1 \%$
$(60.1,80.5)$
$103 / 194=53.1 \%$
$(45.8,60.2)$
$39.3 \%$

$(31.6,47.7)$

$81.1 \%$

(73.0, 87.3)

\begin{tabular}{ll}
$\begin{array}{l}\text { B-RST } \\
(95 \% \text { Cl) }\end{array}$ & $P$ value $^{\text {a }}$ \\
\hline $\begin{array}{l}78 / 83=94.0 \% \\
(86.5,98.0)\end{array}$ & $<0.0001$ \\
$57 / 194=29.4 \%$ & \\
$(23.1,36.3)$ & $<0.0001$ \\
$36.2 \%$ & \\
$(29.9,43.1)$ & NA \\
$91.9 \%$ & NA \\
$(81.5,97.0)$ &
\end{tabular}

Mut mutation, PPV positive predictive value, NPV negative predictive value, NA, not applicable

Plus and minus symbols stand for "positive" and "negative", respectively

a McNemar's chi-square test

no difference in the number of patients who screened negative -average risk between the two versions.

Of 83 mutation-positive pedigrees, 59 screened positive on $\mathrm{B}^{-R_{S T} \mathrm{~T}^{\mathrm{Tm}}}$ 2.0. The most common family history parameter identified in those who screened negative on B-RST ${ }^{\mathrm{m}} 2.0$ $(n=24)$ was a single individual with breast cancer under the age of 40. Adjusting the algorithm to include breast cancer under the age of 45 (current NCCN guidelines) did not increase the sensitivity. The addition of pancreatic cancer, prostate cancer, and cousins also did not significantly affect the sensitivity. Because of this-and to maintain the simplicity of the tool-these parameters were not included in the final algorithm, despite their being part of the current NCCN criteria. $^{15}$

Table 2 shows the validation measures for B-RST ${ }^{\mathrm{mi}} 3.0$ compared with 2.0. Algorithmic changes made to B-RST ${ }^{\text {mo }} 2.0$ increased the sensitivity against BRCA1/2 mutation analysis from 71.1 to $94.0 \%(P<0.0001)$. As expected, the specificity of version 3.0 was significantly lower than that of 2.0 $(P<0.0001)$. Minimal differences in the PPVs were identified. While the NPV of B-RST ${ }^{\mathrm{m} x} 3.0$ was 10 percentage points higher than 2.0, the 95\% confidence intervals overlapped. Predictive values should be interpreted with caution given this was a selected high-risk population.

The mean BRCAPRO probability of B-RST 2.0 false positives was $8.25 \%$ (SD 12.19), compared with $6.12 \%$ (SD 10.61) for B-RST ${ }^{\text {mi }} 3.0$ - a difference that was not statistically significant. The mean BRCAPRO probability of true negatives was 1.48\% (SD 9.20) for B-RST ${ }^{\mathrm{mox}} 2.0$ and 1.13\% (SD 9.21) for $B-R^{2} T^{\mathrm{m}}$ 3.0, and these values were also not significantly different. The mean BRCAPRO probabilities of mutationnegative subjects who screened positive vs. negative differed significantly for both version $2.0 \quad(P<0.0001)$ and 3.0 $(P=0.001)$. Of the five subjects who were false negatives on ${\mathrm{B}-\mathrm{RST}^{\mathrm{m}} \mathrm{m}}^{\mathrm{m}}$ 3.0, the mean BRCAPRO probability was $1.03 \%$ (range $0.12-1.9 \%$ ).

\section{DISCUSSION}

Despite calls for population-based BRCA1/2 testing, there remains a need to assist primary care providers in identifying women at risk for HBOC for referral to cancer genetics services. B-RST ${ }^{\mathrm{mm}}$ was endorsed by the USPSTF for this purpose in 2014. ${ }^{9}$ Previous versions were validated against a $B R C A 1 / 2$ mutation probability of $\geq 10 \%$ - a threshold that is now less frequently considered a minimum for offering genetic testing. ${ }^{10-13,20}$ Utilizing pedigrees of individuals undergoing genetic counseling and BRCA1/2 testing, we modified B-RST ${ }^{\mathrm{m} m}$ to maximize the sensitivity for pathogenic mutations. The revised version, B-RST ${ }^{\mathrm{m} x}$ 3.0, demonstrated superiority over the 2.0 version in sensitivity ( 94 vs. $71 \%$ ). While the specificity with respect to $B R C A 1 / 2$ mutations was lower, the mean BRCAPRO probability of the B-RST ${ }^{\mathrm{m}} 3.0$ false positives was $6.12 \%$, all met 2014 NCCN criteria, and all had been offered genetic testing. Thus, the specificity of B-RST ${ }^{\mathrm{mm}} 3.0$ can be considered high with regards to identifying individuals who should receive cancer genetic counseling (its primary purpose). Maximizing sensitivity over specificity is further justified by the fact that a majority of "false positives" will remain at some increased risk for breast cancer that may lead to alterations in medical management.

The NCCN Guidelines ${ }^{\circledR}$ for $\mathrm{HBOC}$ are widely used to justify referral for genetic evaluation and testing. However, these guidelines are relatively complicated and preclude quick screening by primary care providers. The NCCN criteria are based on specific personal and family cancer parameters rather than $B R C A 1 / 2$ mutation probabilities. A recent study evaluating the NCCN testing criteria reported BRCA1/2 mutations in $3 \%$ of breast cancer patients meeting only one criterion, similar to that found in unselected breast cancer patients. ${ }^{20}$ The authors noted that the NCCN criteria for risk evaluation (genetic counseling) are looser and utility is unclear. ${ }^{20}$ Ease of use and consideration of limited resources for follow-up suggest that tools such as B-RST ${ }^{\mathrm{Tw}}$ may be more appropriate for screening in primary care settings.

There are limitations of this study that deserve to be mentioned. Although a large number of pedigrees were evaluated, an even larger number would provide the opportunity to further refine the tool and increase its sensitivity. Furthermore, this was a retrospective chart review. Prospective evaluation of ${\mathrm{B}-\mathrm{RST}^{\mathrm{m}}}^{\mathrm{m}} 3.0$ in an unselected 
population is needed. In addition, the landscape of genetic testing for breast and ovarian cancer risk has changed, with large cancer gene panels now performed on individuals with less suggestive family histories. Whether B-RST 3.0 in its current form is relevant to non-BRCA1/2 mutations remains unknown. We are completing an implementation study of BRST $^{\mathrm{m}} \quad 3.0$ administered prospectively in mammography settings. One goal of this project is to determine the predictive value of $B-R^{2} T^{m} 3.0$ in identifying individuals with pathogenic mutations in BRCA1/2 as well as other hereditary cancer genes.

We also plan to evaluate changes in medical management recommendations among subjects who did not undergo testing, or were mutation negative.

B-RST $^{\mathrm{Tm}} 3.0$ is a sensitive and useful tool to screen for individuals who should be referred for HBOC genetic counseling. An advantage of B-RST ${ }^{\mathrm{mm}} 3.0$ is that it remains simple enough for most patients to self-administer, and could easily be incorporated into routine physicals. B-RST ${ }^{\mathrm{m}}$ 3.0 is accessible on the internet (www.brcagenescreen.org), and available for incorporation into electronic health records and mammography software. The use of screening tools such as B-RST ${ }^{\text {tw }} 3.0$ on a broader population basis has the potential to increase the identification of individuals and families impacted by BRCA1/2 mutations. This will expand opportunities for reductions in associated cancer morbidity and mortality, while avoiding the potential cost and logistical issues associated with BRCA1/2 population testing.

\section{ACKNOWLEDGEMENTS}

This work was funded in part by a grant from the Brenda Nease Breast Cancer Research Fund, Glenn Family Breast Center, Winship Cancer Institute of Emory University.

\section{DISCLOSURE}

The B-RST ${ }^{\mathrm{TM}}$ is the intellectual property of Emory University School of Medicine. C.B., the developer of B-RSTTM, receives compensation with respect to executed licensing agreements.

\section{REFERENCES}

1. Anglian Breast Cancer Study Group Prevalence and penetrance of BRCA1 and BRCA2 mutations in a population-based series of breast cancer cases. Br J Cancer. 2000;83:1301-8.
2. Maxwell KN, Domchek SM, Nathanson KL, Robson ME. Population frequency of germline BRCA1/2 mutations. I Clin Oncol. 2016;34:4183-5.

3. Rebbeck TR, Friebel T, Lynch HR, et al. Bilateral prophylactic mastectomy reduces breast cancer risk in $B R C A 1$ and $B R C A 2$ mutation carriers: the PROSE Study Group. J Clin Oncol. 2004;22:1055-62.

4. Domchek $S M$, Friebel TM, Singer CF, et al. Association of risk-reducing surgery in BRCA1 or BRCA2 mutation carriers with cancer risk and mortality. JAMA. 2010;304:967-75.

5. Finch AP, Lubinski J, Moller $\mathrm{P}$, et al. Impact of oophorectomy on cancer incidence and mortality in women with a BRCA1 or BRCA2 mutation. J Clin Oncol. 2014;32:1547-53.

6. Drohan B, Roche CA, Cusack JC, Hughes KS. Hereditary breast and ovarian cancer and other hereditary syndromes: using technology to identify carriers. Ann Surg Oncol. 2012;19:1732-7.

7. Childers $C P$, Childers KK, Maggard-Gibbons M, Mackino J. National estimates of genetic testing in women with a history of breast or ovarian cancer. J Clin Oncol. 2017;35:3800-6.

8. United States Preventive Services Task Force Genetic risk assessment and BRCA mutation testing for breast and ovarian cancer susceptibility: recommendation statement. Ann Intern Med. 2005;143:355-61.

9. Moyer VA. Risk assessment, genetic counseling, and genetic testing for BRCA-related cancer in women: U.S. Preventive Services Task Force recommendation statement. Ann Intern Med. 2014;160:271-81.

10. Bellcross C, Lemke A, Pape L, Tess A, Mesiner L. Evaluation of a breast/ ovarian cancer genetics referral screening tool in a mammography population. Genet Med. 2009;11:783-9.

11. Bellcross C. Further development and evaluation of a breast/ovarian cancer genetics referral screening tool. Genet Med. 2010;12:240.

12. Robson ME, Bradbury AR, Domchek SM, et al. American Society of Clinical Oncology policy statement update: genetic and genomic testing for cancer susceptibility. J Clin Oncol. 2015;33:3660-7.

13. Committee on Practice Bulletins-Gynecology, Committee on Genetics, Society of Gynecologic Oncology Practice bulletin no 182: hereditary breast and ovarian cancer syndrome. Obstet Gynecol. 2017;130: e110-26.

14. Daly MB, Pilarski R, Axilbund JE, et al. Genetic/familial high-risk assessment: breast and ovarian, version 1.2014. I Natl Compr Canc Netw. 2014;12:1326-38

15. National Comprehensive Cancer Network [Internet]. Clinical Practice Guidelines in Oncology: Genetic/Familial High-Risk Assessment: Breast and Ovarian. Version 1.2018. Available from: http://www.nccn.org. Accessed 3 Oct 2017.

16. King MC, Levy-Lahad E, Lahad A. Population-based screening for $B R C A 1$ and BRCA2: 2014 Lasker Award. JAMA. 2014:312:1091-2.

17. Yurgelun $M B$, Hiller $E$, Garber JE. Population-wide screening for germline BRCA1 and BRCA2 mutations: too much of a good thing? I Clin Oncol. 2015:33:3092-5.

18. Cintolo-Gonzalez JA, Braun D, Blackford AL, et al. Breast cancer risk models: a comprehensive overview of existing models, validation, and clinical applications. Breast Cancer Res Treat. 2017;164:263-84.

19. U.T. Southwestern Medical Center at Dallas and The BayesMendal Group [Internet]. CancerGene. Available from: http://www4.utsouthwestern. edu/breasthealth/cagene/. Accessed 25 June 2015.

20. Cropper C, Woodson A, Arun B, et al. Evaluating the NCCN clinical criteria for recommending $B R C A 1$ and $B R C A 2$ genetic testing in patients with breast cancer. J Natl Compr Canc Netw. 2017;15:797-803. 